\title{
El cotidiano del cuidado de la salud de las familias pobres: un abordaje de la fenomenología social
}

\author{
María Arcaya-Moncada ${ }^{1, a, b}$, Carlos Tello- Pompa ${ }^{2, a, c}$, Rosane Nitschke- Gonçalves ${ }^{3, c}$
}

\section{RESUMEN}

Objetivos: comprender el cotidiano del cuidado de la salud de las familias en situación de pobreza. Material y métodos: el abordaje teórico-metodológico fue la fenomenología social, que permitió comprender el cotidiano del cuidado de la salud de las familias, que se inserta en una trama de acciones y relaciones intersubjetivas entre los miembros de la familia, derivadas de la convivencia y el compartir experiencias en el día a día y de las que se obtienen las señales para interpretar el cotidiano. Diseño cualitativo, descriptivo e interpretativo. El escenario fue una comunidad urbano marginada, los sujetos de estudio fueron diez madres de familia e hijos en edad escolar. Se utilizó la entrevista a profundidad. Resultados: revelan la comprensión del cotidiano del cuidado de la salud de las familias pobres. Emergen las categorías: la vida cotidiana compartida marcada por lo vivido en el cuidado de los hijos, los quehaceres del hogar y el trabajo. Afrontando la adversidad, con sus límites y fuerzas, en el cotidiano del cuidado de la salud de la familia. Conclusiones: se destaca el cotidiano del cuidado de la salud, develando el típico vivido y los motivos "para y porqué".

PALABRAS CLAVE: Cotidiano, cuidado de la salud de las familias, Fenomenología Social.

\section{The daily health care of poor families: a social approach of phenomenology}

\section{SUMMARY}

Objectives: to understand the daily health care of families in poverty from the perspective of the mother and her children, from a social phenomenology approach. Material and Methods: the theoretical-methodological approach was the social phenomenology, to understand the daily care of the health of the families, that it inserts into a intersubjective web of social relationships that exists among family members, arising from living together and sharing the day to day experiences from which signals are obtained to interpret it. A qualitative descriptive and interpretative design. The location was a marginalized urban community. The subjects of the study were 10 mothers and their school-age children. In the study it was used the in-depth interview. Results: the results reveal the everyday understanding of health care for poor families. It arises the categories: the daily routine marked by the care of the children, housekeeping and work, dealing with the adversity with their limits and strength in the daily care of the family health. Conclusions: among the final considerations it is highlighted the importance of immersing into the health care and the unveiling the daily living. of the everyday health considering the "for and why" motives.

KEYWORDS: Everyday, health care for families, Social Phenomenology.

Universidad Nacional Mayor de San Marcos. Lima, Perú.

Universidad Nacional de Trujillo. Trujillo, Perú.

Universidad Santa Catarina. Florianópolis, Brasil.

Profesora Principal ; ${ }^{\mathrm{b}}$ Magister en Enfermería ; ${ }^{\mathrm{c}}$ Doctor en Enfermería 


\section{INTRODUCCIÓN}

La privación económica como reflejo de la realidad social, plantea en la familia una serie de problemas relacionados con la obtención de alimentos, asignación de prioridades para los gastos, incertidumbre sobre el futuro, frustración, niveles altos de estrés que conllevan a conductas violentas con los niños, menos calidez y empatía con sus hijos. Lo que puede afectar de manera adversa el desarrollo, la salud y bienestar de sus miembros. La mayor o menor probabilidad de muerte y enfermedad está en función de factores como el estrato socioeconómico, la condición de ruralismo, el género y el nivel educativo en que se encuentren las personas y las comunidades. Para comprender el comportamiento de las familias pobres es necesario ahondar en el conocimiento de los espacios sociales donde se crean y recrean las relaciones primarias entre los individuos y el papel central que estos juegan en la lucha por la sobrevivencia cotidiana. La visión romántica e idealizada del hogar como el refugio por excelencia ante las adversidades de la vida, ha quedado atrás para la mayoría de los estudiosos sobre el tema. La familia es el escenario social privilegiado para el cultivo de los afectos y los odios más profundos y perdurables a lo largo de la existencia. Las relaciones familiares reflejan en su complejidad" (1).

Vivir en familia implica necesariamente el enfrentamiento cotidiano con la desigualdad y el conflicto abierto o encubierto entre los diferentes miembros. En este espacio social confluyen intereses opuestos que suelen llevar a ganancias para unos y pérdidas para otros. Las posibilidades de negociación están altamente determinadas por la posición jerárquica y de género que ocupan los miembros del hogar. Las decisiones sobre los modos y formas de vivir en familia no pueden ser vistas como producto de una participación equitativa e incondicionada; en la familia, cada miembro se juega día a día el pase hacia la pertenencia o hacia la exclusión, sea emocional o física.

Por otro lado; una de las mayores atribuciones de la familia es el cuidado de la salud de sus miembros durante los ciclos de salud-enfermedad e incluyen tanto las enseñanzas de las prácticas de cuidado a la salud como la garantía del soporte social durante la salud y la enfermedad. Aunado a ello se espera que la familia de hoy atienda las necesidades interacciónales de sus miembros, como son: amor, intimidad, desarrollo de auto aceptación y cuidado, entre otros. Este "cuidado cotidiano", que se estructura en gran medida en el seno de la familia, llega a constituirse en el cuerpo de conocimiento y práctica de cada familia, que estructura su propio sistema de cuidado, el que da cuenta de todo lo que la familia va aprehendiendo, creando y produciendo en relación con mecanismos de protección de su propia existencia, generando acciones que van orientadas a dar respuestas a requerimientos de las dimensiones física, psíquica, social, espiritual y cultural. Este sistema de cuidado que llega a estructurar y sustentar la familia, con la participación de todos sus integrantes, en interacción con su medio, le asigna características particulares, reflejadas en sus hábitos, costumbres, rutinas; donde se conjugan elementos teóricos y prácticas heredadas y adquiridas. Este recurso, propio de cada familia, se construye a partir de su vivencia con el mundo cotidiano. Cada familia define el valor del cuidado, como también de la salud. Son dos conceptos que, en el ámbito de la misma, se encuentran vinculados y se influyen recíprocamente. Así, como ella inicia a cada uno de sus integrantes en el desarrollo de acciones de cuidado, lo hace a partir de la concepción de salud que tiene y al valor que le asigna. Las acciones que la persona y familia generan para cuidarse, está relacionada con la forma en que se percibe así misma y a su mundo, de acuerdo al significado asignado, especialmente a salud y bienestar. Las acciones que desarrolla son a partir de su propia percepción de su realidad y recursos disponibles (2).

De lo anterior se deduce que la familia cumple un papel trascendente en el cuidado de la salud de sus miembros, siendo la madre la responsable por la salud de la familia. Eso indica la necesidad de profundizar en el papel desempeñado por cada miembro en la estructura familiar, reflexionar sobre los aspectos en la organización familiar, es decir, la forma en que la familia divide las responsabilidades entre sus miembros para cumplir el papel de educadora, proveedora, cuidadora y socializadora de los hijos.

El entendimiento de la pobreza a través del estudio de las subjetividades es reconocer las formas en que se expresa, se significa, se siente y se enfrenta la vida cuando se vive en condiciones de pobreza extrema en las grandes ciudades (3).

En este marco, es importante estudiar el cotidiano del cuidado de la salud, desde la perspectiva de la madre cuidadora y de los hijos, en su contexto familiar donde se dan las situaciones de cuidado de la salud, así como las significaciones construidas colectivamente a partir de las acciones sociales que ellas desarrollan para cuidar su salud y la interacción social en el mundo de vida cotidiana, considerando a la pobreza como un factor determinante 
que influencia directamente en el cotidiano y calidad de vida de las familias. Comprender sus motivaciones, la construcción del sentido que dan a sus acciones del cuidado que conforman el típico vivido en el cuidado de la salud familiar.

Estos cuestionamientos orientaron a la pregunta del estudio de investigación:

¿Cómo es el cotidiano del cuidado de la salud en las familias en situación de pobreza desde la perspectiva de la madre e hijos?

Se plantearon los siguientes objetivos: comprender el cotidiano del cuidado de la salud de las familias en situación de pobreza, en la perspectiva de la madre e hijo (a), desde el abordaje de la Fenomenología Social. Conocer el cotidiano del cuidado de la salud de las familias en situación de pobreza, desde la perspectiva de la madre e hijo (a). Aprender el típico vivido del cotidiano del cuidado de la salud de las familias pobres.

En el presente estudio se utilizó el referencial teórico metodológico de la fenomenología Social de Schütz que permite captar la realidad social, situar a las personas en su actitud natural y traer el mundo de vida cotidiana en el que ellos se sitúan en intersubjetividad con sus pares y permite la captación del otro como único en su individualidad. El ser humano se relaciona y confiere significado a su acción como ser consciente (4). Así, se pretende aprender, conocer y comprender las intenciones y motivaciones de la madre en la experiencia de cuidar la salud de sus familias en un contexto donde se dan las relaciones, interacciones y vivencias compartidas entre los miembros del grupo social, fundamentado en la esencia del fenómeno y en la subjetividad.

El objetivo del presente trabajo fue cmprender el cotidiano del cuidado de la salud de las familias en situación de pobreza.

\section{MATERIAL Y MÉTODOS}

Esta investigación fue desarrollada tomando como base el abordaje cualitativo, en la elección del tipo de estudio se consideró la naturaleza de su objeto, es decir el cotidiano de las familias, la misma que requiere ser comprendida, interpretada en sus significados desde la perspectiva de los actores sociales envueltos en el contexto de lo vivido. El estudio es de naturaleza cualitativa apoyado en el referencial teórico metodológico de Schütz. El diseño es descriptivo e interpretativo.
El método fenomenológico social, el que parte del mundo conocido, del cual hace un análisis descriptivo del fenómeno como tal, en base a las experiencias compartidas, y de las experiencias intersubjetivas se obtienen las señales, indicaciones para interpretar la diversidad de símbolos. A partir de allí, es posible interpretar los procesos y estructuras sociales. La metodología es el camino del pensamiento y la práctica ejercida en el abordaje de la realidad (5).

Los sujetos de estudio fueron trece madres de familias y el hijo mayor. Se utilizó como instrumento una guía semiestructurada de preguntas abiertas Para complementar los registros de información se tomaron las notas de campo que comprendió el registro de las observaciones realizadas del contexto familiar, reflexiones, emociones, percepciones del propio investigador durante la entrevista. Se tuvieron en cuenta los principios bioéticos y el código internacional de Helsinski que rige la investigación científica, para lo cual se aplicó el consentimiento informado. Se aplicó la técnica de saturación de datos para determinar el tamaño de la muestra.

\section{RESULTADOS}

Este estudio proviene de las vivencias de las familias en su cotidiano cuidando la salud de sus miembros, emergiendo las categorías temáticas concretas de lo vivido que se presentan a continuación:

I Categoría: La vida cotidiana compartida marcada por lo vivido en el cuidado de los hijos, los quehaceres del hogar y el trabajo.

\section{Algunos testimonios:}

"Para cuidar de mis hijos, me levanto temprano, los dias que me toca trabajar, para mi es estresante ..., lo estresante es el tiempo de estar alistándome para salir al trabajo porque tengo que alistar a mi hija para el colegio, preparar la lonchera, dejar en el colegio, ir a mi trabajo, lo estresante de todo el día es que tengo salir al trabajo y venir casa para seguir atendiendo a mi bebita" Lirio.

"Tengo mi mamá de ochenta y cinco años vive conmigo y mi mamá bueno me ayuda, pero también tengo que ver por ella tiene 85 años, en las tardes vendo productos cosméticos, mi día es estresante para mi" Clavel.

"Siempre lavo los utensilios para que no vaya a infectarse, tenemos silo, hay que estar desinfectando, le digo que se abrigue, si se enferma hace gasto” Jazmín. 
"Hija; yo trabajo para que nada te falte nada, me estoy sacrificando para ti, tú estás creciendo hay más gastos, las cosas que han pasado, tu papá no da nada (dinero)"

II Categoría: Afrontando la adversidad, límites y en el cotidiano del cuidado de la salud de la familia.

Emergiendo algunas subcategorias que se presentan a continuación:

Manifestaciones del cuidar con emocionalidad, afecto, esperanzasy frustraciones.

"Una vez mi hijo Daniel se enfermó, mi esposo no tenía trabajo, la plata no le alcanzaba, yo no tenía dinero para llevarlo a la posta, tenía seguro pero no había dinero, mi hermana me prestó". Alheli

"Estoy pendiente de ellos, tengo una hija delicada de salud, las medicinas están caras, debemos ver la manera, voy hacer un actividad para recaudar fondos, si compro la medicina". (...). "Quisiera salir y estar mejor pero si no hay dinero no se puede (lagrimas... silencio), algún día las cosas serán diferentes,"Amapola.

\section{Darle duro, tenemos que salir de abajo}

"Yo quisiera salir y estar mejor pero si no hay dinero no se puede, (lagrimas...silencio), algún día las cosas serán diferentes, el tiempo pasa y las cosas van a mejorar.

"Soy fuerte me levanto temprano, ahi estoy, para darle duro, a las circunstancias, seguir adelante, trabajando de alguna manera uno sobresale" Amapola.

La fuerza principal que da sentido a su vida cotidiana en el cuidado de la familia se evidencia en la expresión " $L o$ hago con todo amor". "Los cuido porque los quiero, los amo, deseo superarme en lo que esté a mi alcance, para que ellos se sientan bien y cómodos, es el amor a mi familia la que me impulsa a seguir adelante.....” Camelia.

\section{La espiritualidad presente en el cotidiano de las familias}

"Soy católica y tengo fe en Jesús" Amapola.

\section{Redes de apoyo social en la familia}

"Mi hijo Daniel se enfermó, mi esposo no tenía trabajo, la plata no le alcanzaba, yo no tenía dinero para llevarlo a la posta, tenía seguro pero no tenía dinero, mi hermana me prestó. Lo dejaba a mi hijo al cuidado de mi hermana porque yo trabajaba". Alheli.
III Categoría: Los motivos "para y porqué" en el cotidiano del cuidado de la familia.

"Los cuido porque los quiero, los amo, deseo superarme en lo que esté a mi alcance, para que ellos se sientan bien y cómodos, es el amor a mi familia la que me impulsa,". Camelia.

"No quisiera que mi hija termine como yo, en un lugar como este, no sea una mediocre como yo, que no tenga que agachar la cabeza, que mi hija sea alguien importante en la vida, para que no sufra, lo hago, con cariño y amor" Camelia.

\section{DISCUSIÓN}

Schütz, se interesa sobre todo por la comprensión de la acción social como el sentido que el actor asigna a su acción, es decir el sentido que su acción tiene para él, piensa en la interpretación subjetiva del sentido, como una tipificación del mundo del sentido común, la manera concreta en que los hombres interpretan en la vida diaria, su propia conducta y la de los demás es decir la interpretación subjetiva del sentido, así como todo el problema de la comprensión interpretativa (6). Se presentan las categorías:

\section{El cotidiano marcado por lo vivido en el cuidado de los hijos, los quehaceres del hogar y el trabajo.}

El cotidiano del cuidado de la salud de las familias , es un mundo natural, intersubjetivo y compartido con los demás, se revela en el cuidado de los hijos, los quehaceres del hogar y el trabajo las experiencias y la realidad de lo vivido por las madres de familia del AAHH Héroes del Cenepa, se considera como el ámbito en el que actúan e interactúan y se complementan los miembros de la familia, está marcado por actividades de la vida diaria, los quehaceres propios del hogar que se materializan en cuanto se dirigen al cuidado del otro. Esta categoría convergen con los hallazgos de otros autores que estudian el cotidiano desde la perspectiva fenomenológica filosófica (7). El "mundo cotidiano" es entendido como ámbito de acción social, para Schütz, el "mundo de la vida" tiene su propia existencia, su propia lógica y su propia organización, es decir, el "mundo de la vida" es independiente del actor. Sin embargo, los actores comparten sus interpretaciones, sus experiencias, sus vivencias y construyen la vida de manera coparticipativa (8). 
El mundo cotidiano se revela en el cuidado de los hijos, los quehaceres del hogar y el trabajo las experiencias y la realidad de lo vivido por las madres de familia, se considera como el ámbito en el que actúan e interactúan y se complementan los miembros de la familia, está marcado por los quehaceres propios del hogar que se materializan en cuanto se dirigen al cuidado del otro. En el universo simbológico de los pobres existe una división complementaria entre hombres y mujeres en el universo de la familia, la casa es identificada con la mujer y la familia con el hombre, casa y familia como mujer y hombre constituyen un par complementario más jerárquico. Cualquier discusión sobre el cuidado es remetida inmediatamente al "universo femenino" pues desde la infancia hay un claro imaginario colectivo de que el cuidado esté presente en la postura de las niñas (9). El Cotidiano del cuidado es compartido con los hijos quienes colaboran con los quehaceres del hogar, está marcado por las expectativas de recibir amor, protección, seguridad y afecto de los padres, la que se objetiva en actos de acompañamiento, el estar allí en el momento que lo requieran, compartir momentos de conversación en familia corrobora el principio de la familia definida como la permanente búsqueda de espacios de aproximación, de involucramiento con los otros explica (10).

\section{Afrontando la adversidad, con sus límites y fuerzas.}

Mi mundo cotidiano no es mi mundo privado, sino más bien mi mundo intersubjetivo compartido por mis semejantes experimentado e interpretado por otros $(11-12)$. El "mundo cotidiano" es entendido como ámbito de acción social, para Schütz, el "mundo de la vida" tiene su propia existencia, su propia lógica y su propia organización, es decir, el "mundo de la vida" es independiente del actor. Los actores comparten sus interpretaciones y cuando realizan ese ejercicio comparten sus experiencias, comparten sus vivencias y construyen la vida de manera coparticipativa (7).

A partir de sus propios significados, ellos perciben y afrontan el reto del cuidado de la salud de la familia, son parte de la lucha por la vida, caracterizada por manifestaciones y actitudes de afecto, como el desvelo, preocupación, sacrificios, desesperanza, deseos, expectativas, angustia cuando el hijo enferma y la falta de medios económicos, por otro lado surge manifestaciones de afecto, amor, colaboración y la esperanza de un futuro mejor para sus hijos.

\section{Las fuerzas para afrontar la adversidad}

El vivir el día a día implica una búsqueda emprendida consigo mismo, con los otros y con el mundo. Al identificar sus fuerzas las personas se colocan con una expresión de fuerza de querer vivir, la que emerge de la potencia subterránea (13).

Para superar las dificultades y adversidades que atentan contra el cuidado y su bienestar ellas desarrollan una "fuerza" que en muchos casos se evidencia por la perseverancia para vencer desafíos del cotidiano, el amor a sus hijos, el valor y la esperanza, el deseo de superación fe en Dios, las familias desarrollan una serie de estrategias y mecanismos que buscan de manera intencionada atenuar los efectos de las crisis y consecuente deterioro en las condiciones de vida.

Las familias son conscientes que para enfrentar la adversidad solo trabajando se sobresale, por lo deciden seguir adelante, hay un sentimiento de culpa en estas madres, los niños y jóvenes son criados en un contexto de familia muy frágil, los padres trabajando, padres separados, la falta de comunicación, se construye así muy poco el sentido de vida.

En los testimonios, se visualiza la Fe en Dios, la esperanza y la creencia centrada en la religiosidad, las expresiones denotan una carga de espiritualidad presente en el cotidiano de las familias. La espiritualidad es aquello que produce en el ser humano un cambio interior, (...) el objetivo fundamental es el ser humano como un centro a partir del cual todo se liga y religa, permitiendo un encuentro con la totalidad de la existencia. se trata de una dimensión presente en cada ser humano que se revela a través de la capacidad de diálogo consigo mismo y con el propio corazón y se traduce en el amor, en la sensibilidad, en la escucha del otro, y en cuidado con todo y con todos (14).

El individuo tiene un interés eminentemente práctico de dominar y modificar su mundo de vida cotidiana a fin de alcanzar sus propósitos de vida (15). En este sentido las familias tienen expectativas, esperanzas, deseos e ilusiones que esperan alcanzar a futuro mejor, hacia la que direccionan todos sus esfuerzos. Las fuerzas presentes en su cotidiano tienen ciertas similitudes con los resultados del estudio de Argollo reporta que las fuerzas que demostraron las familias brasileñas fueron la persistencia; la fuerza de voluntad; la fe; la esperanza; el conocimiento de la enfermedad o la alteración de la salud individual y familiar; los vínculos familiares y sociales (16). 


\section{Los motivos "para y porqué" compartidos en el cotidiano del cuidado de la salud de las familias.}

Los "motivos para” están constituidos por las motivaciones para cuidar la salud de sus familias, en otras palabras se constituyen en los significados que tienen sus actos. Los que concuerdan con sus expectativas, deseos y proyectos de superación personal y de su familia, seguido por el deseo de sentirse bien, estar sanos, cómodos y satisfechos consigo mismo y con los demás, estos motivos reafirman el amor que sienten por la familia. La preocupación por la educación de los hijos emerge como un "motivo porque", que constituye la fuerza, una aspiración, a la vez un reto para la familia, la misma tiene como propósito lograr el progreso con ello la posibilidad de salir de la pobreza no teniendo que pasar por privaciones y lo vivido por los padres.

El cotidiano del cuidado de la salud de las familias pobres, se representan en la figura 1.

\section{CONCLUSIONES}

El cotidiano del cuidado de las familias pobres, está representado por todo lo que hacen, todo lo que viven, todo lo que tienen que pasar para cuidar la salud de su familia, la manera cómo interactúan en su medio para cuidar de sí y de los otros miembros, identificando sus dificultades y fuerzas, la comprensión del cotidiano permitirá que los profesionales de Enfermería pueden estar en mejores condiciones de brindar cuidados socialmente pertinentes, recrear el modo de atender a sus necesidades y expectativas, rescatando sus fuerzas para enfrentar los límites del cotidiano.

El cotidiano del cuidado de la salud de la familia esta traspasado por una red de significados que representan la acción de cuidar la salud de la familia, confiere un sentimiento de pertenencia a un ambiente familiar, donde las relaciones cotidianas se expresan con armonía, realizaciones, esperanzas, cariño, cuidado, responsabilidad, compromiso, empatía ,preocupación, entre otras.

El cotidiano del cuidado de la salud de las familias pobresesta demarcado por la "lucha del día a día" por salir adelante, los límites u obstáculos son superados con lucha y valor para enfrentar las dificultades.

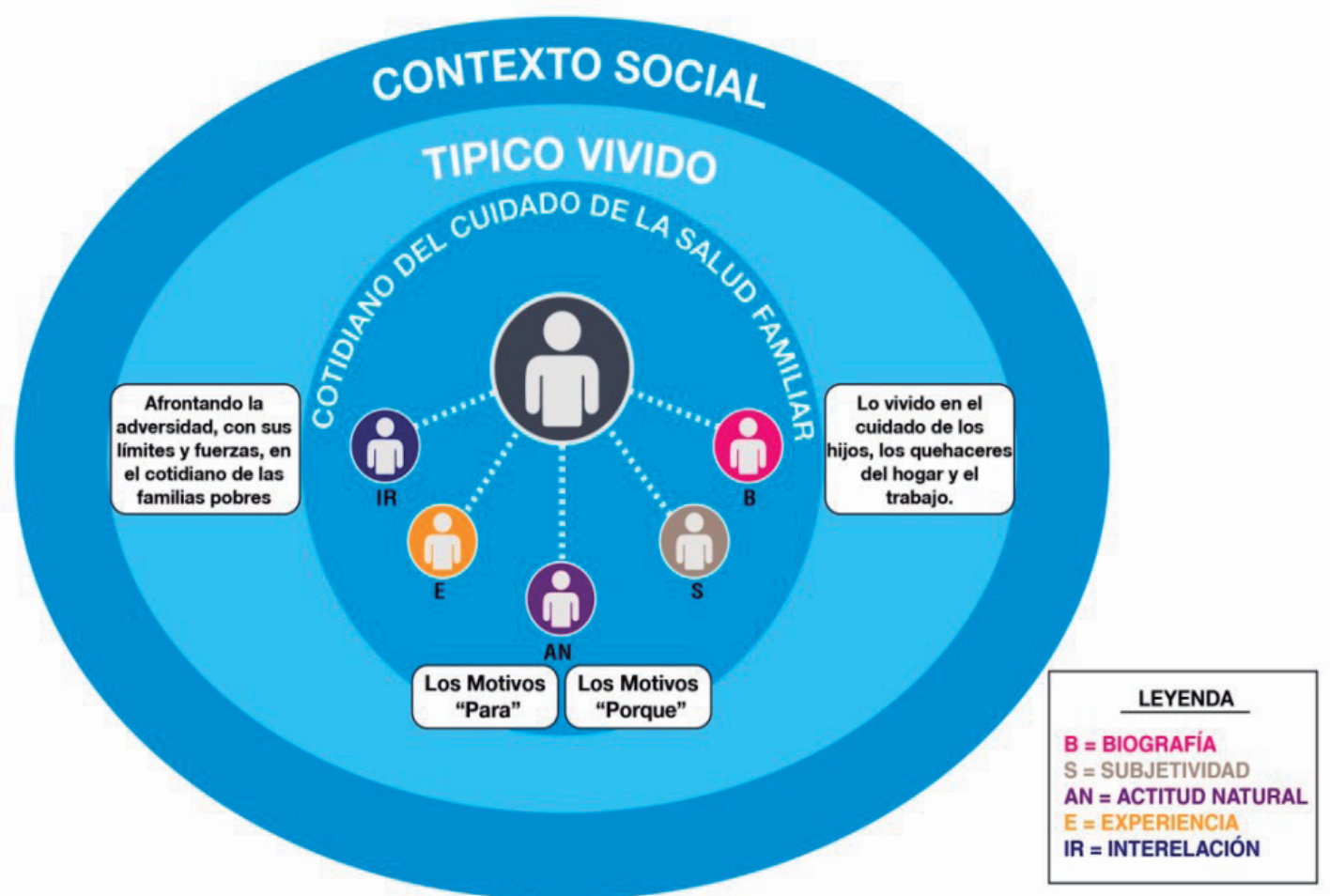

FIGURA Nº1 - Esquema conceptual sobre el Cotidiano del Cuidado de la Salud 


\section{REFERENCIAS BIBLIOGRÁFICAS.}

1. Kaztman R. Activos y estructuras de oportunidades: Estudios sobre las raíces de la vulnerabilidad social en Uruguay. Montevideo: PNUD / CEPAL; 1999.

2. Delgado J. El cuidado cotidiano y la salud de la familia. Familia. Saúde Desenv. 2001;3(1): 21-25.

3. Enríquez-Rosas R. El crisol de la pobreza: mujeres, subjetividades, emociones y redes sociales. Tlaquepaque, Jalisco, Mexico: ITESO; 2009. p. 165 -167.

4. Schütz A. Estudios sobre teoría social. Buenos Aires: Amorrortu; 1974. pp. 22- 25, 43-45.

5. Minayo M. Pesquisa social: teoria, método e criatividade. Brasil: Editora Voces. 2002.p. 54-56.

6. Natanson M. El problema de la realidad social. Buenos Aires: Amorrotu Editores; 1995. p. 327.

7. Tello C. El cotidiano de vida y de salud de mujeres peruanas de una comunidad rural. Tesis de Doutorado em Enfermagem. Rio de Janeiro: Escola de Enfermagem Anna Nery, Universidade Federal do Rio de Janeiro; 2,003.

8. Schütz A. El problema de la realidad social. Buenos Aires: Amorrortu Editores; 1995.p. 16-31, 20, 39, 280.

9. Rojas A, Faller V, editores. Familia, Redes, Laços e Políticas Públicas. 4ta edic. Sao Paulo: Cortes Editora. 2008. pp.2829, 33-34.

10. Bustamante E. Enfermería Familiar. Principios del Cuidado Familiar a partir del saber in común de las familias. Trujillo, Perú: Universidad Nacional de Trujillo; 2004. p. 57-87.

11. Schütz A. El problema de la realidad social. 2da edición. Buenos Aires: Amorrortu Editores. 2003. pp..19,20, 16-39, 280 .

12. Schütz A. La construcción significativa del mundo Social. Buenos Aires: Ediciones Paidos. 1993.p. 45-50.

13. Maffesoli M. O tempo das tribos: o declínio do individualismo nas sociedades. Brasil: Editora Forense Universitária; 1988.
14. Boff L. Espiritualidade: um caminho de transformação. Rio de Janeiro: Sextante de massa. Rio de Janeiro: Forence; 1998. p. 47.

15. Schütz A. El problema de la realidad social. Buenos Aires: Amorrortu Editores. 1995.p. 16-31, 20, 39, 280.

16. Argôllo S. O quotidiano com seus limites e forças para o ser saudável: um encontro da enfermagem com a potência para contornar a violência no dia-a-dia. Tesis Doctoral. Florianópolis, Brasil: Universidade Federal de Santa Catarina; 2007.

\section{Correspondencia:}

María Josefa Arcaya Moncada.

Correspondencia: marcayam@yahoo.com

Fecha de Recepción: 13 de junio del 2016. Fecha de aceptación: 29 de junio del 2016. 